\title{
Curiosity is associated with enhanced tonic firing in dorsal anterior cingulate cortex
}

Maya Zhe Wang and Benjamin Yost Hayden

Contact Information: Maya Zhe Wang Department of Neuroscience \& Center for Magnetic Resonance Research University of Minnesota, Minneapolis MN 55455 Email: mayawangz@gmail.com

\section{Keywords}

Observing behavior, entropy, anterior cingulate cortex, orbitofrontal cortex, curiosity

\section{Acknowledgements:}

We thank Tommy Blanchard for help in designing the task, and Marc Mancarella and Meghan Castagno for assistance in data collection. We appreciate close help from Ethan Bromberg-Martin for help in designing the task, and developing some of the analysis approaches we used here. This research was supported by an NIH R01(DA038106) to BYH. 
ABSTRACT

38 an important driver of information-seeking and curiosity. To gain insight into its neural

39 basis, we recorded responses of single neurons in dorsal anterior cingulate cortex (dACC)

40 while rhesus macaques performed a task that induces and quantifies demand for

41 information. We find that enhanced firing rates in $\mathrm{dACC}$ before the start of a trial predict

42 a stronger bias towards information-seeking choices. Following choices of uninformative

43 options, firing rates are tonically enhanced until information is delivered. The level of

44 enhancement observed is correlated on a trial-by-trial basis with the value assigned to the

45 prospective information. Finally, variation in this tone is positively correlated with

46 receptiveness to new information, as inferred by preference changes on subsequent trials.

47 These patterns are not observed in a complementary dataset collected in orbitofrontal

48 cortex (OFC), suggesting these effects reflect at least somewhat anatomically localized

49 processing. 


\section{INTRODUCTION}

51 Ignorance is not always bliss. A decision-maker who is uncertain about the

52 outcomes of their potential actions and choices may have a desire to probe the

53 environment for information that can provide the missing knowledge. Indeed, decision-

54 makers may gain utility from doing so, even if the information is neutral or bad (Kidd

55 and Hayden, 2016; White et al., 2019). This fact has motivated scholars to propose that

56 curiosity is motivated in part by an information gap, ego dystonic discrepancy between

57 current and desired information (Golman \& Loewenstein, 2015; Gottlieb et al., 2013;

58 Kang et al., 2009; Loewenstein, 1994). In this view, lack of information is a special drive

59 state that can be sated by obtaining information. The information gap is the central

60 theoretical structure linking curiosity to psychology and ultimately to neuroscience

61 (Golman \& Loewenstein, 2018; Gottlieb \& Oudeyer, 2018; Kidd \& Hayden, 2016;

62 Marvin \& Shohamy, 2016; van Lieshout et al., 2018).

63 Despite its value in motivating psychological hypotheses, the neuronal basis of

64 the information gap remains to be identified (Cervera et al., 2020). We hypothesized that

65 the brain computes and represents the demand for information within a circumscribed

66 circuit. Several factors motivated us to hypothesize that the dorsal anterior cingulate

67 cortex (dACC) would be one such region. The dACC is associated with monitoring both

68 cognitive and visceral (i.e. basal drive state) variables (Heilbronner \& Hayden, 2016a;

69 Morecraft \& Van Hoesen, 1998). At least one study has linked activity in dACC to

70 curiosity (Jepma et al., 2012). Neurons in dACC also track - and drive demand for -

71 counterfactual information, suggesting the region may monitor current information gap, 
72 and drive information-seeking decisions (Hayden, et al., 2009). Moreover, enhanced

73 hemodynamic activity in this region is associated with enhanced control, with

74 specification of control, and with exploratory processes in foraging, which have some

75 heuristically similarity to information-seeking (Kolling et al., 2012; Shenhav et al., 2013;

76 Shenhav et al., 2017; Smith et al., 2019; Heilbronner and Hayden, 2016). Finally, activity

77 in this region is directly associated with information-seeking processes, with curiosity per

78 se (e.g. Jepma et al., 2012). Given these facts we hypothesized that dACC neurons would

79 track current level of information gap.

80 Here we made use of the curiosity tradeoff task that we developed previously

81 (Blanchard et al., 2015). This task is based a version of the observing task designed for

82 macaques (Bromberg-Martin \& Hikosaka, 2009; Roper, 1999). On each trial, subjects

83 choose between two gambles with different stakes and then wait 2.25 seconds until they

84 are rewarded. One option provides information about the resolution of the gamble

85 immediately; the other option maintains the mystery for the delay period. Monkeys are

86 reliably information-seeking in this task, meaning they will sacrifice a small amount of

87 water to obtain advance (Blanchard et al., 2015). We have proposed that this task satisfies

88 an operational definition of curiosity (Wang \& Hayden, 2019). Specifically, we believe

89 that information-seeking choices in this task reflect a demand for information reflective

90 of an information gap. Moreover, we believe that choice of an uninformative option leads

91 to a state in which information is lacking and therefore maintains an information gap. In a

92 previous study, we reported the responses of neurons in orbitofrontal cortex (OFC)

93 during this task, although we did not examine either of these epochs (Blanchard et al., 
bioRxiv preprint doi: https://doi.org/10.1101/2020.05.25.115139; this version posted May 25, 2020. The copyright holder for this preprint (which was not certified by peer review) is the author/funder. All rights reserved. No reuse allowed without permission.

94 2015). For the present study, we compared this dataset to a second dataset, collected at

95 the same time as the first but not previously analyzed, recorded in dACC.

96 

(tructure of the task (above) and different possible stimuli (below). B. Coronal section of subject $\mathrm{H}$ showing the location of recording sites in $\mathrm{dACC}$. C. Behavior of two subjects on standard trials in dACC/OFC datasets (darker/lighter colors). Likelihood of choosing informative option as a function of relative value between the two options. Leftward shift of curves indicates that both subjects preferred the

\section{A}

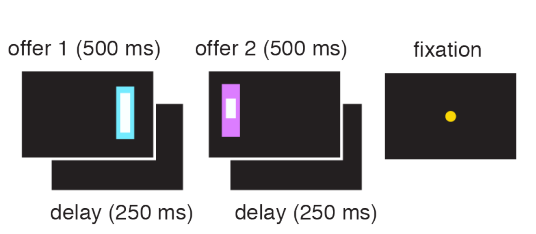

choice

cue and delay

inter-trial interval
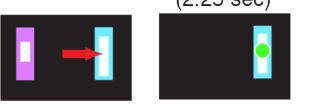

Standard trial stimuli $(70 \%$ of all trials):

Variable probability trial stimuli (30\% of all trials):

cyan bar: informative option

magenta bar: uninformative option

central white bar: water amount for gamble win, $50 \%$ probability

red and green circles (cyan bar): gamble loss and win cues

red and green circles (magenta bar):

uninformative decoys, identified by context

\section{B}

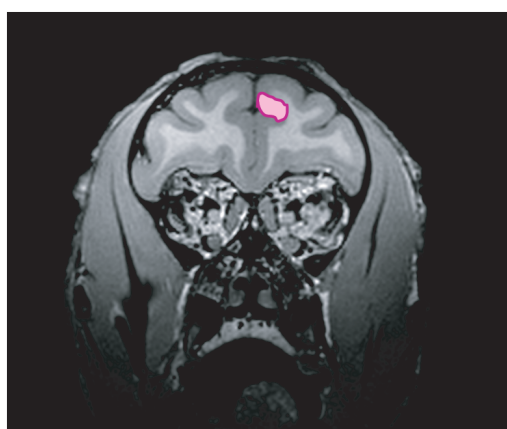

C

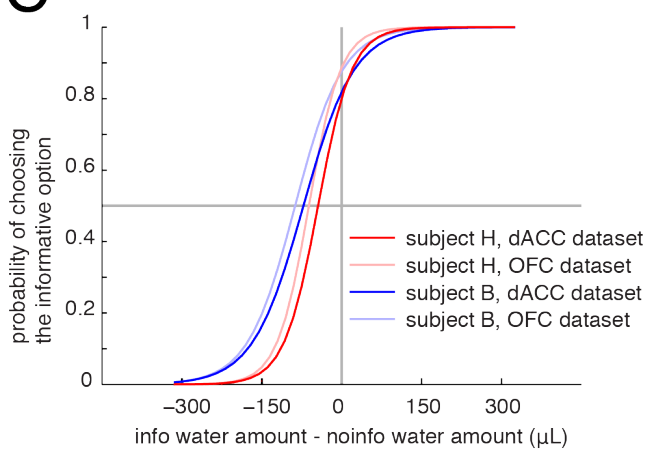

Figure 1. Task, anatomy, and basic behavior. A. Cartoon illustrating the informative option on standard trials.

Behavior: macaques value advance information about gamble outcomes

We used a task we called the curiosity tradeoff task that we developed previously

112 the design of our study). 
114 reward of varying amount (Figure 1). Regardless of choice, any reward is given 2.25

115 seconds later. Behavior of macaques in this task has been described in detail (Blanchard

116 et al., 2015; Bromberg-Martin \& Hikosaka, 2009; Bromberg-Martin, Matsumoto, \&

117 Hikosaka, 2010). Indeed, these two macaques were the same subjects used in our

118 previous study and behavior here is, not surprisingly, nearly identical (Blanchard et al.,

119 2015, Figure 1C). As in our previous study, both subjects preferred informative cues.

120 Subjects B and $\mathrm{H}$ chose the gamble with higher stakes on $78.2 \%$ and $83.0 \%$ of trials (both

121 are greater than chance, $\mathrm{p}<0.0001$, binomial test). Subjects B and H chose the more

122 informative option on $67.8 \%$ and $69.4 \%$ of trials respectively (both $\mathrm{p}<0.0001$, binomial

123 test). When the two options had equal stakes, both subjects preferred information (B:

$12478.8 \%, \mathrm{H}: 78.1 \%)$. Indifference points (Methods) for the two subjects were $76 \mu \mathrm{l}(\mathrm{B})$ and

$12551 \mu \mathrm{l}(\mathrm{H})$. This indifference point identifies the subjective value of information.

$126 \quad$ Variable probability trials (30\% of trials): These trials were not used in our

127 previous study and were introduced here as an additional control. On $30 \%$ of trials

128 (randomly interleaved), subjects chose between two uninformative options that have the

129 same stakes (225 uL juice). The probability was either $25 \%, 50 \%$, or $70 \%$ and was the

130 same for both offers on the same trial. On these trials, subjects chose the left and right

131 option roughly equally (subject B: 55.1\% left; subject H: 49.8\% left). Any observed

132 left/right bias did not depend on probability (regression of left choice against the three

133 probability conditions, subject $\mathrm{B}: \mathrm{p}=0.44$, subject $\mathrm{H}: \mathrm{p}=0.18$ ). 


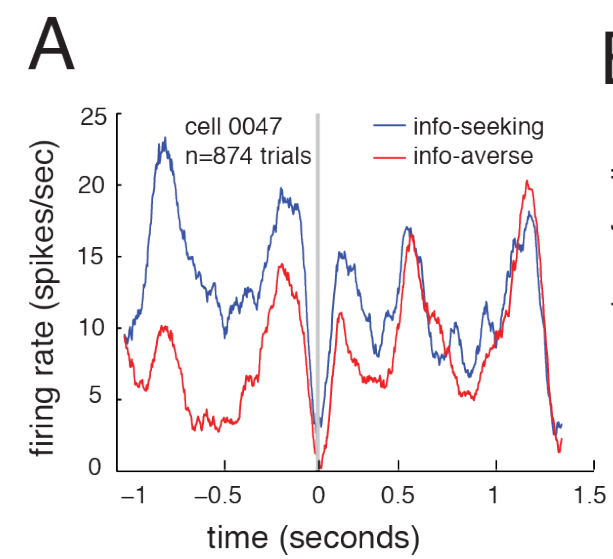

Figure 2. Pre-trial correlation between demand for information and firing rates in dACC neurons. A. Responses of an example neuron showing higher firing rates on info-seeking trials vs. info-averse trials (these categories are determined by average behavior, see main text). B. Histogram of pre-trial differences between info-seeking and info-averse trials. Neurons with individually significant effects are shown in black.

\section{Enhanced pre-trial activity in dACC predicts information-seeking choices}

We recorded responses of 151 single neurons in $\mathrm{dACC}(\mathrm{n}=88$ in subject $\mathrm{B}$ and $\mathrm{n}=63$ in subject $\mathrm{H}$ ). We collected an average 551 trials per cell, and a minimum of 500 trials. We reasoned that if demand for information reflects a drive state, it would have neuronal signatures before trial onset. We therefore considered the $500 \mathrm{~ms}$ period immediately preceding the presentation of the first offer. We divided all trials into two categories, (1) ones that were more information-seeking than average, (2) ones that were less information-seeking than average. These categories were defined in terms of the average subjective value the subject placed on information as inferred by the choice made during the task. Many trials could not be assigned to a category and were therefore excluded from this analysis (Methods). 
153 For the example neuron shown in Figure 2A, pre-trial activity was higher on

154 relatively information-seeking trials ( $\mathrm{p}=0.004$ Student's $\mathrm{t}$-test). Responses of $27.2 \%$ of

155 neurons $(n=41 / 151)$ differentiated the two trial types (this proportion is significant,

$156 \mathrm{p}<0.001$, binomial test, Figure 2B). Of these, $75.6 \%(\mathrm{n}=31 / 41)$ showed enhanced firing

157 (this proportion is significant, $\mathrm{p}=0.0015$, binomial test). Responses of $26.4 \%$ of neurons

$158(n=40 / 151)$ differentiated information-seeking trials relative to neutral trials (as

159 determined by $t$-test, this proportion is significant, $p<0.001$, binomial test). Of these

160 neurons, $70.0 \%(\mathrm{n}=28 / 40)$ were enhanced (this proportion is significantly different from

$1610.5, \mathrm{p}=0.0166$, binomial test). Thus, increased pre-trial firing predicts information-

162 seeking choices. Indeed, the average ensemble firing rate for all neurons (including non-

163 significantly modulated ones) was 0.71 spikes/sec greater preceding information-seeking

164 trials than neutral trials and $0.42 \mathrm{spikes} / \mathrm{sec}$ lower on information-averse trials than on

165 neutral ones (both these differences are significant, $\mathrm{p}<0.001$, $\mathrm{t}$-test). These numbers

166 represent a relatively high proportion ( $17.32 \%$ and $10.24 \%$, respectively) of the baseline

167 pre-trial firing rate (that is, $4.1 \mathrm{spikes} / \mathrm{sec}$ ). 

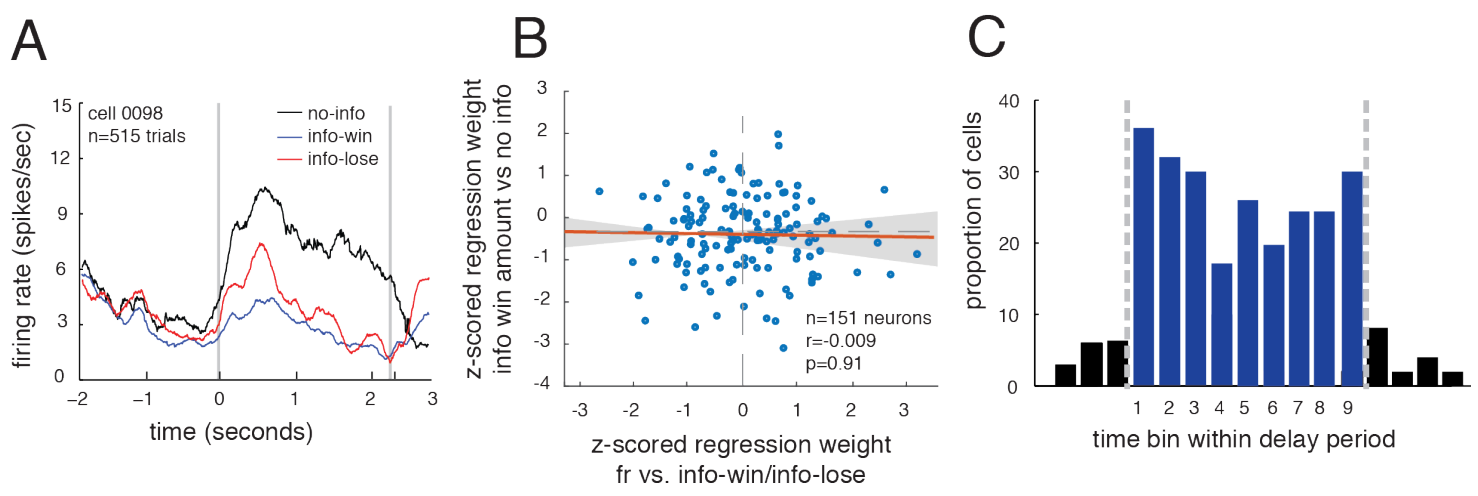

168

169

170

171

172

173

174

175

176

177

178

179

180

181

182

183

184

185

186

187

188

189
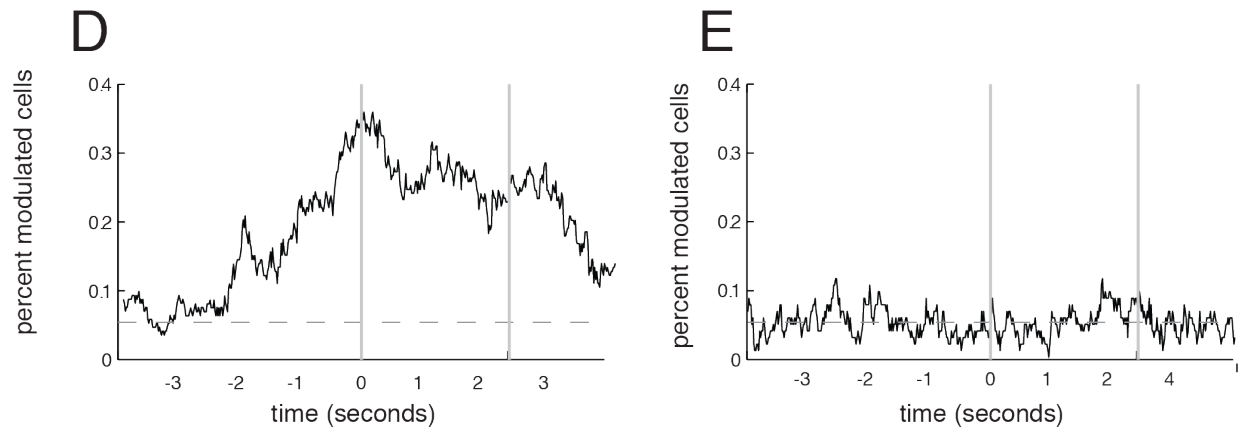

Figure 3. Delay period response modulation for no-info trials. Time 0 in A, $D$, and $E$ indicates the start of delay period. A. Responses of an example neuron on trials in which no upcoming reward information is given during the delay (noinfo trials, black) and trials in which this information is given (info trials, blue and red). Responses on no-info trials are systematically enhanced, a pattern that is common in the population. B. Scatter plot of regression weights for info vs. no info trials (y-axis) against info-win vs. info-lose (x-axis). These variables are not correlated, suggesting that codes for information gap and reward are unrelated. C. We divided data into nine time bins and found significant modulation in each one, suggesting, on no-info trials, the modulation is sustained across the delay period. D. Plot of proportion of cells significantly modulated by info vs. no-info status, using a sliding $500 \mathrm{~ms}$ window. Horizontal dashed line indicates chance level (i.e., 5\%). E. Plot of proportion of cells significantly modulated by the winand lose-related cues on no-info trials (when they are non-predictive). We see no measurable effect.

\section{Informational uncertainty tonically enhances firing rates in dACC}

On trials in which the subject chose the no-info option (no-info trials), subjects proceeded to enter a state of temporally extended uncertainty. During this period, the subject did not know whether a reward would occur for 2.25 seconds. We next asked how 
190 neurons would respond to this sustained lack of uncertainty resolution. We reasoned that

191 if uncertainty has no special implications, then the firing rate may resemble a weighted

192 average of the firing rates associated with the two possible contrapositive outcomes

193 (learning that a large/no reward is impending). On the other hand, if the status of lacking

194 information in this task is somehow special, it may lead to a firing rate outside the range

195 of the other two, and, in particular, systematic enhancement in firing across the long

196 period the uncertainty is maintained.

197 For a typical neuron (Figure 3A), responses on no-info trials are enhanced (2.9

198 spikes/sec and 3.2 spikes/sec, $\mathrm{p}<0.01$ in both cases, Student's t-test). In our entire sample,

199 firing rate on no-info trials was different from the average firing rate on both types of info

200 trials in a substantial number of neurons $(46.3 \%, \mathrm{n}=70 / 151, \mathrm{p}<0.001$, binomial test $)$. This

201 modulation appears to last the entire waiting period. We divided the 2.25 second waiting

202 period into nine equal $250 \mathrm{~ms}$ time bins. In all nine bins, a significant proportion of cells

203 encoded the variables for info vs. no info. Even the bin with the lowest proportion had

$20418 \%, \mathrm{n}=28$ cells, which is greater than chance $(\mathrm{p}<0.001$, binomial test $)$.

205 We next asked whether neurons that showed enhancement in one of these epochs

206 were more likely to be the ones that showed enhancement in another. (That is, whether

207 these effects reflect a sustained enhancement in some neurons, or periodic short bursting

208 in more neurons). We reasoned that if the same set of cells was involved in signaling

209 information from one bin to another, then we would see a positive correlation in their

210 unsigned regression weights (i.e. absolute value of regression weights, see Azab \&

211 Hayden, 2017 for details). For every pair of bins ( $n=72$ comparisons, i.e. 9 time bins $x 8$ 
212 other time bins), the cells involved were more overlapping than chance (correlation was

213 significant, average $\mathrm{r}=0.29, \mathrm{p}<0.05$ in all individual cases $)$.

214 We next considered the average effect of informational status on aggregate (grand

215 average) firing rate. We found that the average firing rate on all no-info trials (8.22

216 spikes/sec) for all neurons (including non-significantly modulated ones) was greater than

217 on info-trials (5.97 spikes/sec; this difference is significant, $\mathrm{p}<0.001$, Student's t-test).

218 The population of significantly modulated cells was positively biased, meaning more

219 individual neurons showed an increase in firing than showed a decrease $(74.3 \%, \mathrm{n}=52 / 70$,

$220 \mathrm{p}<0.001$, binomial test).

221 Note that this average positive deflection is unlikely to reflect a sustained version

222 of the bias the predicted information-seeking choices (see previous section). That bias led

223 to greater firing before info trials, whereas the delay period modulation showed the

224 reverse pattern. Thus, any firing rate hysteresis would presumably have reduced our

225 measured effects, not spurred a false positive.

\section{Delay period enhancement is greater on high information-demand trials}

229 (willingness to pay) rises with stakes of the chosen option (Blanchard et al., 2015). These

230 results indicate that demand for information is higher on higher stakes trials (i.e. trials on

231 which the subjects are in suspense about a higher valued gamble). Overall, responses of

$232 \quad 21.9 \%$ of cells $(n=33 / 151)$ were modulated by the stakes during the no-info delay period;

233 the majority $(72.7 \%, n=24 / 33)$ showed an enhancement (this bias is significant, 
$234 \mathrm{p}=0.0135$, binomial test). Indeed, the average firing of the population was greater in the

235 top stakes tercile than in the bottom stakes tercile (difference in the entire population,

2361.32 spikes/sec, $\mathrm{p}<0.001$, Student's t test). Nonetheless, the firing rate in the bottom

237 tercile was greater than responses in either info-win or info-lose conditions (difference in

238 the entire population, 1.91 spikes $/ \mathrm{sec}, \mathrm{p}<0.01$, Student's t test).
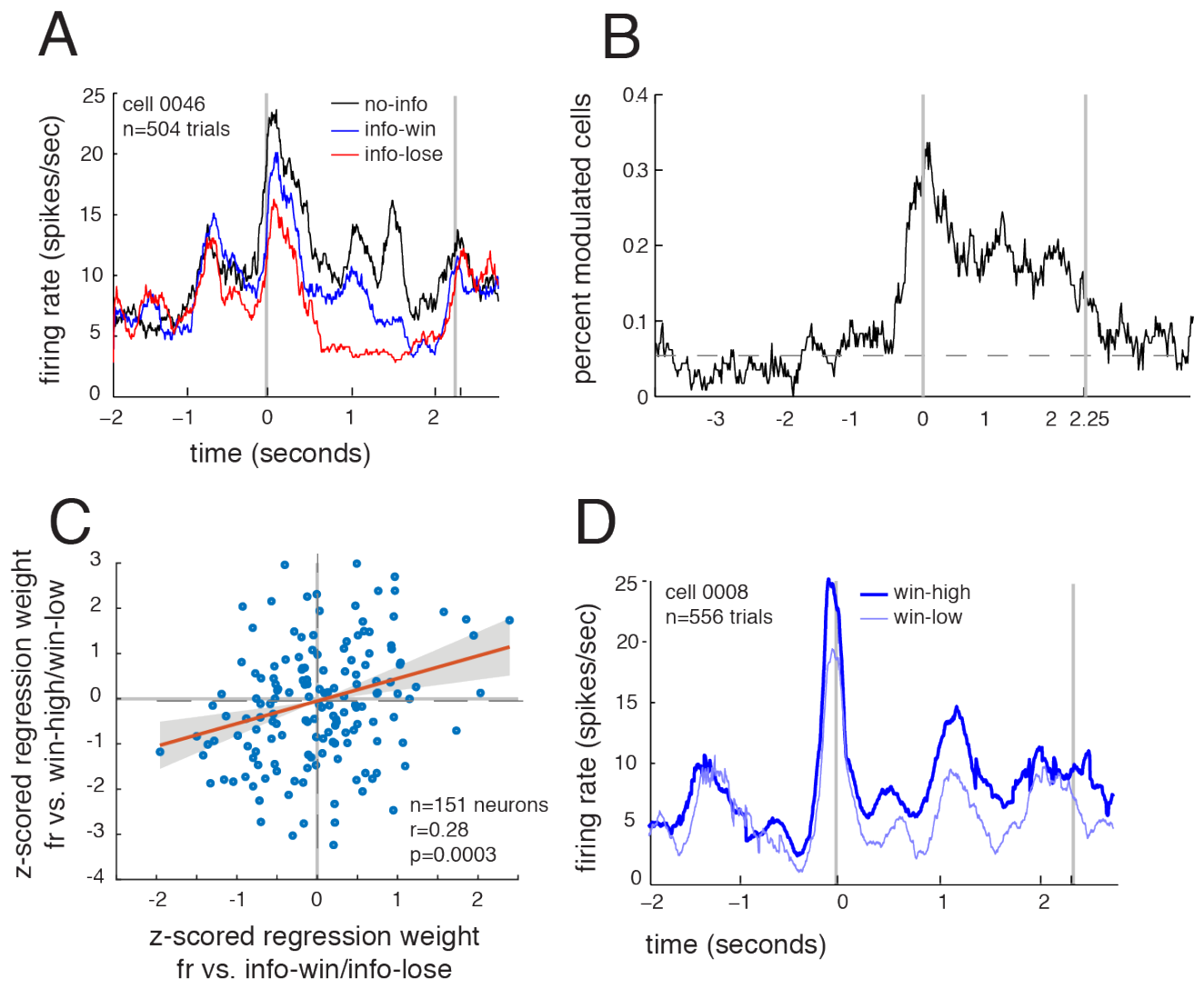

Figure 4. Neuronal encoding of upcoming rewards. A. Responses of an example neuron during the delay period (starting at time 0 on the graph) on infowin (blue) and info-lose (red) trials. Info-win and info-lose trials are significantly different throughout the course of the delay (0 to 2.25 seconds). Firing rates on no-info trials (black) are also shown, for reference. B. Proportion of cells whose responses significantly modulated by the difference between info-win and infolose using a sliding $500 \mathrm{~ms}$ window. Horizontal dashed line indicates chance level (i.e., 5\%). C. Scatter plot showing regression weights for info-win-high vs. info-win-low (y-axis) against info-win / info-lose (x-axis). The positive correlation indicates that dACC neurons use correlated codes for the two value variables. $\mathbf{D}$. 
250 Responses of an example cell to info-win trials when the stakes are high (thick 251 line) and low (thin line).

252

253 volume anticipated. For the neurons in Figure 4D, the average firing rate was higher on

268 info-win trials with larger than average rewards (thick line) than with smaller than average rewards (thin line, difference, 2.1 spikes/sec, $\mathrm{p}=0.009$ ). On info-win trials,

271 (regression of firing rate against size of anticipated reward). This bias was also not

272 directional ( 19 positive and 26 negative, $\mathrm{p}=0.37$, binomial test). The neural coding 273 pattern, namely strength and direction, used by dACC neurons for the win-loss bias was 
274 closely correlated with that the reward volume effect, suggesting that this effect reflects a

275 generic reward encoding (correlation of tuning indices for the two dimensions, $\mathrm{r}=0.31$,

$276 \mathrm{p}<0.001)$. This correlation indicates that, within $\mathrm{dACC}$, there is a general code for

277 anticipated reward - that is win vs. loss uses the same coding format as amount won on

278 win trials. In any case, this result suggests that the lack of correlation between codes for

279 information gap and for reward vs. no reward (see above and Figure 2A) is not simply

280 and artifact of noise. (And indeed, that correlation, $r=-0.02$ is significantly lower than this

281 one, Fisher r-to-z, $\mathrm{z}=-2.97$, two-tailed $\mathrm{p}=0.003$, see below).

A

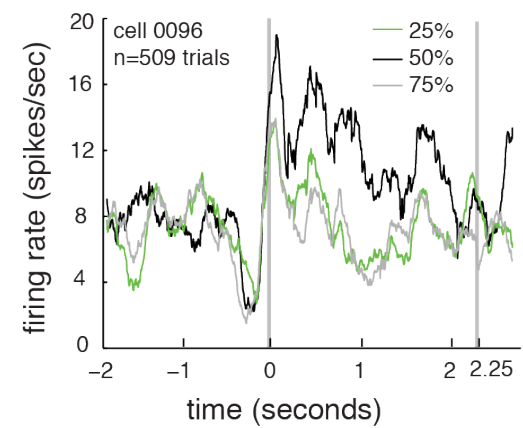

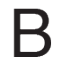

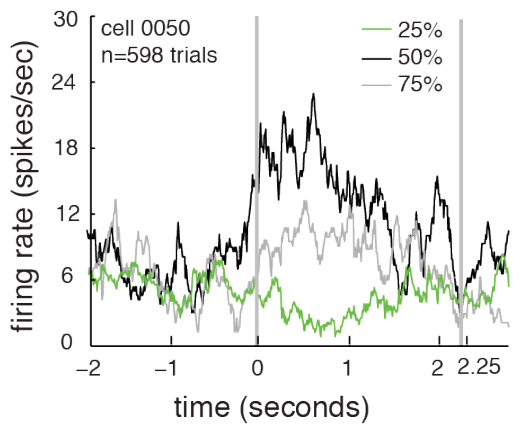

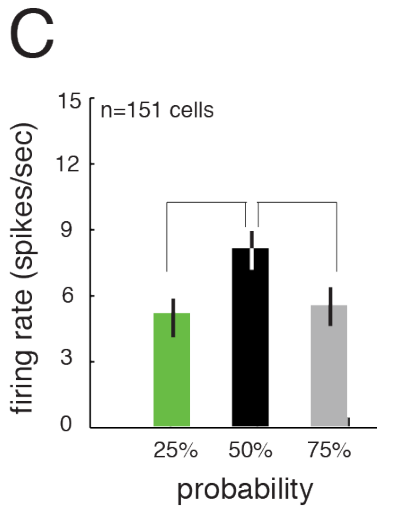

Figure 5. Data from variable probability trials. A. and B. Responses of two example neurons on variable probability trials. For both neurons, responses were greater on $50 \%$ trials than they were on $25 \%$ and $75 \%$ trials, suggesting the neurons is more concerned with entropy than it is with expected value. Time 0 refers to the start of the delay period. C. Grand average of responses for the population on variability probability trials. Responses of the ensemble are greater on $50 \%$ than on either $25 \%$ or $75 \%$ trials.

\section{Controlling for confounds with reward and arousal in dACC}


296 consequence of reward or reward anticipation coding, then we should see the neural

297 coding pattern for information gap and reward related variables to be significantly

298 similar. Otherwise, information gap evokes a differentiable pattern than do reward related

299 variables in dACC. To test this idea, we therefore computed an info-gap coefficient (the

300 linear term of the regression coefficient for firing rate against no-info vs info) and two

301 reward indices for each neuron, one related to info-win vs. info-lose (win-lose coefficient)

302 and one related to the size of the anticipated reward on the info-win trials (win-amount

303 coefficient, see Methods).

304 The correlation between the info-gap coefficient and the two reward indices was

305 not significantly different from zero in either case (win-lose coefficient: $r=-0.02, p=0.59$;

306 win amount coefficient: $\mathrm{r}=-0.033, \mathrm{p}=0.36$ ). Because this lack of effect is difficult to

307 interpret - it may reflect noise - we next estimated sample noise using a previously

308 developed cross-validation technique, Blanchard et al., 2015). The correlations we

309 observed within sample for info-gap coefficient with the two reward coefficients were

310 both significantly greater than zero $(r=0.67$ and $r=0.38$, respectively, $p<0.01$ in both

311 cases). They were also significantly greater than the observed correlations (differences

312 were $\mathrm{p}<0.001$ in both cases, bootstrap test), indicating that noise was not a limiting factor

313 and indicating that our observed correlation was significantly less than the value we

314 would have observed had the true correlation been 1.0. These results suggest that

315 information gap and arousal (both reward-related coefficients) evoke unrelated neural

316 response patterns and thus the effect of information gap cannot be simply explained away

317 by arousal. 
It is also worth noting that the modulation observed on no-info trials does not

319 appear to reflect the low level features of the stimuli; on info and no-info trials, the same

320 two cues were presented, but they had either reward-predictive or reward-irrelevant

321 meaning, depending on context (Figure 3E). On no-info trials, dACC neurons did not

322 encode the color of the decoy cue ( $5.3 \%$ of cells did so, $n=8 / 151, p=0.85)$.

323 To gain additional perspective on the potential confound with arousal, we

324 included a new trial type. On variable probability trials (30\% of all trials), subjects chose

325 between identical offers. These trials had either $25 \%, 50 \%$, and $75 \%$ stakes and a

326 medium reward. Responses of two example neurons are shown in Figures 5A and 5B.

327 These neurons showed greater firing on 50\% trials than on the other two trial types.

328 Overall, $52.3 \%$ of neurons $(n=79 / 151)$ showed a significant difference for the conditions

329 (ANOVA test on individual neurons).

330 The example neurons are typical - we found that on these trials, neurons

331 differentiated $25 \%$ from $50 \%$ (difference for all neurons: 3.46 spikes/sec, $\mathrm{p}<0.001$ ), and

$33250 \%$ from $75 \%$ (difference for all neurons: 2.68 spikes/sec, $\mathrm{p}<0.001$ ), although they did

333 not differentiate $25 \%$ from $75 \%$ (difference: 0.39 spikes $/ \mathrm{sec}, \mathrm{p}=0.34$ ). Note that these

334 analyses reflect control for multiple comparisons. This pattern suggests that neurons

335 encode entropy (sometimes called uncertainty), rather than expected value. In other

336 words, the most parsimonious explanation of the factors driving neural responses is

337 "amount of information available." To formally test this idea, we compared linear and

338 quadratic models; we found that the quadratic model fit better in more of the condition- 
340 Burnham \& Anderson, 2010).
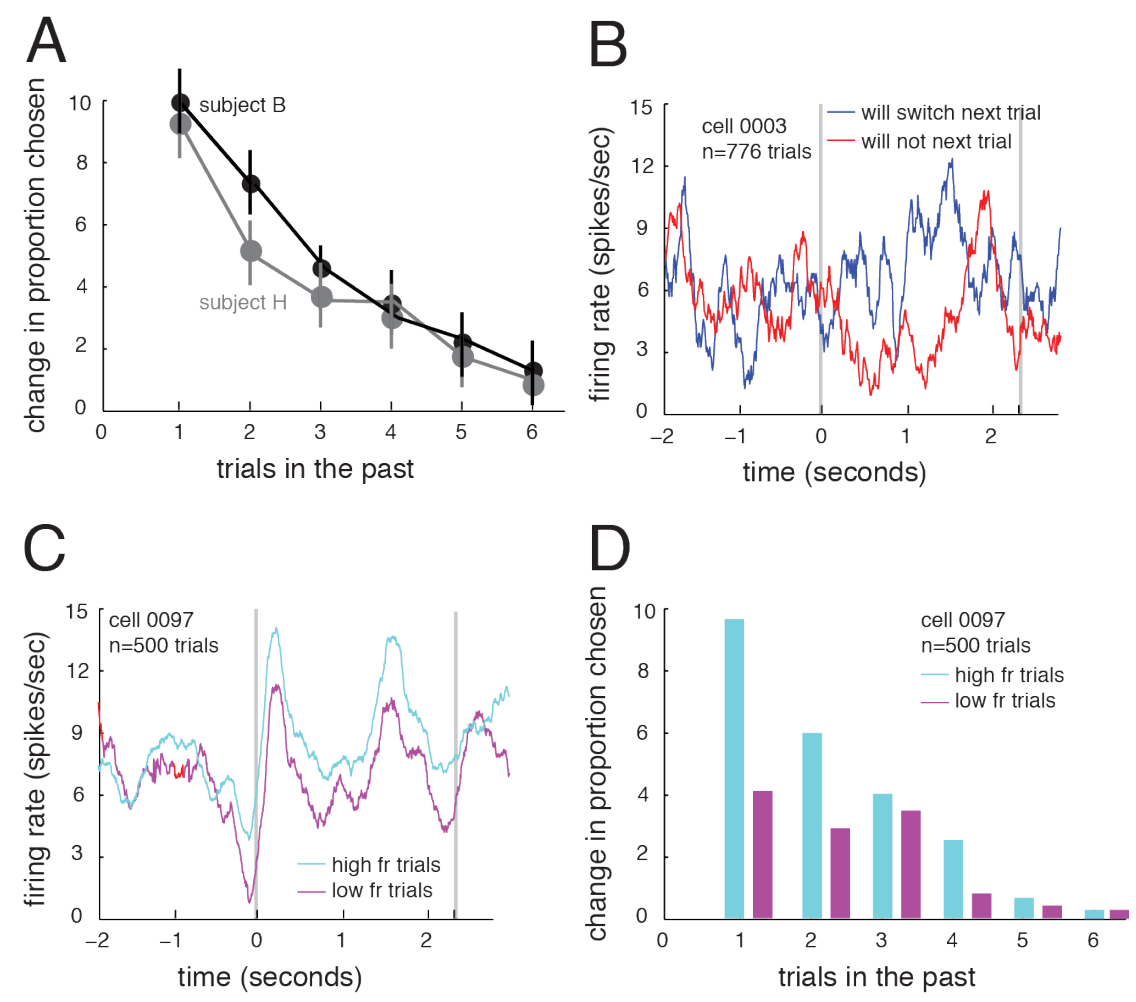

Figure 6. Data related to adjustment and likelihood of changing strategy. 0 point on $\mathrm{X}$ axis in $\mathrm{A}-\mathrm{C}$ reflect the start of delay period. A. Subjects switch sides more often following gambling losses than gambling wins; this effect persists 3-4 trials. B. Plot of firing rate of an example cell showing different firing rates on oninfo trials (i.e. controlling for information status and reward status) separated by whether the subject will switch on the next trial. C. Plot of an example cell in high and low firing rate trials (note that this effect, while significant, is a consequence of our analysis). Time zero indicates start of delay period. D. On higher firing rate trials for the neuron shown in panel B, subjects are more likely to adjust behavior on subsequent trials. 
We wondered if the firing rate enhancement we saw correlates with readiness to

358 learn. We have previously investigated the effects of risky outcomes on behavioral

359 adjustments in some detail (Hayden et al., 2009; Hayden et al., 2011). For present

360 purposes, the key idea is that switching - whether or not it is beneficial - is driven by

361 attention to recent outcomes, so that variability in propensity to switch reflects variability

362 in receptivity to recent outcomes.

363 Here, we find that following wins, subjects are more likely to choose the same

364 side (left vs right). Specifically, relative to losses, on wins, subject B showed a 9.6\%

365 increased likelihood of repeating the rewarded side and subject $\mathrm{H}$ showed a $10.0 \%$

366 increase (these numbers, while small, are significantly greater than $0, p<0.001$, binomial

367 test, Figure 6A). These effects are observable as far out as 4 trials later. Gamble wins

368 changed preference at a statistically significant level for subject B (3.5\% increase,

$369 \mathrm{p}=0.0288)$ and for subject $\mathrm{H}(3.8 \%$ increase, $\mathrm{p}=0.0446)$.

370 We next asked how these trial-to-trial adjustment effects correspond to variations

371 in firing rate. Figure 6B shows the delay period firing rate of an example neuron on no

372 info trials. This neuron showed enhanced firing rate for info-gap and this firing rate

373 predicted choice switch on the next trial. Figure 6C shows the responses of an example

374 neuron on no-info trials, separated into higher and lower than average firing rates, after

375 regressing out stakes. For this neuron, responses were 1.81 spikes/sec higher on higher

376 firing rate trials ( $\mathrm{p}=0.019$, $\mathrm{t}$-test; note that this difference is pre-ordained by the analysis).

377 Figure 6D then shows the adjustment pattern for this session on both trial types. This

378 overall pattern was also observed in the population. Specifically, we performed a linear 
379 regression of firing rate in the window against side switch (binary variable, 1 for switch, -

3801 for no-switch ), including additional factors for stakes and past win/lose. Responses of

$38122.5 \%(n=34 / 151)$ of neurons show a correlation with switching, after regressing out

382 other variables; $76.4 \%$ are positive $(n=26 / 34$, this proportion is significant, $p=0.0029$,

383 binomial test). We also performed a linear regression of firing rate in the window against

384 strategy switch (binary variable), including additional factors for stakes and past

385 win/lose. We find that responses of $12.5 \%$ of neurons $(n=19 / 151)$ show a significant

386 correlation with switching, and that $15 \%$ are positive $(n=15 / 19, p=0.0192)$.

387

388

390

391

392

393

394

395

396

397

398

399

400

\section{Lack of corresponding effects in OFC}

We collected complementary results in a study of the OFC (those data are summarized in Blanchard et al., 2015). In our previous manuscript reporting on that dataset, our analyses focused on responses to offers, whereas here we consider pre-trial and delay period effects. Here, we report new results focusing on the pre-trial and delay period effects in the OFC dataset.

Overall, OFC appears very weakly involved in the aspects of the task that strongly drive dACC responses. In OFC, variability in firing rates pre-trial did not predict information-seeking decisions. Specifically, $7.96 \%$ of neurons ( $\mathrm{n}=9 / 113$ neurons) showed a firing rate correlation with upcoming choice (not significant, $\mathrm{p}=0.1877$, binomial test). This proportion is significantly smaller than the proportion observed in dACC (i.e. $25.8 \%, \mathrm{p}<0.001$, binomial test). The average firing rate before information-seeking trials was not different than the average firing rate before information-averse trials (difference: 
4010.11 spikes/sec, $\mathrm{p}=0.85$, t-test). Uncertainty about upcoming rewards did enhance delay

402 activity in OFC in a significant proportion of neurons, although the proportion was close

403 to threshold ( $9.7 \%$ of cells, $n=11 / 113, p=0.0293$, binomial test $)$. The effect was visible as

404 an increase in firing as in OFC, although the effect is not significant (difference: 0.44

405 spike/sec, $p=0.33)$, and is significantly lower than the difference in dACC $(p<0.001$,

406 Student's t-test).

407 Finally, variation in firing rate in OFC did not predict adjustments in behavior.

408 Specifically, we observed this correlation in $2.65 \%$ of cells $(n=3 / 113)$. This proportion is

409 not significant ( $\mathrm{p}=0.29$, binomial test) and is significantly lower than the proportion

410 observed in $\mathrm{dACC}(\mathrm{p}<0.01$, binomial test). These results together suggest that OFC does

411 not strongly predict information seeking behavior or strategy adjustment after the

412 resolution of epistemic uncertainty. 


\section{DISCUSSION}

414 Curiosity, a drive for non-instrumental information, clearly has multiple possible

415 causes. Here, we asked whether those causes can include mitigating the costs associated

416 with uncertainty. Specifically, we reasoned that remaining in a state of suspense may be

417 aversive in part because it carries some metabolic costs. To test this hypothesis, we

418 examined the responses of single dACC neurons during an information tradeoff task

419 (Blanchard et al., 2015). We find that tonically enhanced firing rates in dACC predict

420 information-seeking on a trial-by-trial basis, a potential neuronal correlate of the that is a

421 hypothesized driver of curiosity. Choice of uninformative options leads to a sustained

422 tonic enhancement in firing that persists until the information is provided. Variability in

423 this enhancement predicts demand for information and sensitivity of the subject to

424 outcome information (as assessed by adjustment behavior). These changes were not

425 observed in OFC, suggesting that our putative enhancements in activity related to

426 uncertainty are at least somewhat anatomically localized. These observations in turn

427 endorse the idea that dACC serves in part to accumulate evidence for purposes of guiding

428 action (Hayden et al., 2011; Hayden and Heilbronner, 2016; Hunt et al., 2018; Kolling et

429 al., 2012).

430 Neurons in many prefrontal regions encode multiple task variables and, typically,

431 neural correlates of task variables show a population-level balance of positive and

432 negative responses. This overall balance likely reflects the fact that positive and negative

433 deflections can both carry information and, because spiking is costly, there are metabolic

434 benefits that accrue to a brain that can keep overall spiking levels low regardless of the 
435 situation. The putative correlates of information gap we introduce here, in contrast, are

436 biased towards the positive direction. The bias towards the positive direction suggests the

437 speculative possibility that encoding these variables imposes metabolic costs on dACC

438 (Laughlin et al., 1998). These costs did not appear to be counteracted by savings in other

439 task epochs or in at least one other brain region, the OFC. If the brain is efficient at

440 managing its own energy budget, it will seek out situations that can reduce spiking. Thus,

441 our results provide tentative evidence consistent with the hypothesis that demand for

442 information in this task reflects a demand for energy efficiency.

443 Why would it be costly to do be in a state of suspense? One possibility is that,

444 when there is information available to learn, the brain's learning systems enter into a state

445 of eligibility, that is, they have the ability to enter into multiple possible knowledge

446 states. Perhaps these knowledge states are low-energy, but the metastable state in which

447 multiple knowledge states are possible is higher energy. Another - not incompatible -

448 possibility is that the brain must enter into a state of enhanced vigilance to monitor

449 information and that the acquisition of that information allows the brain to reduce its

450 vigilance and focus on other tasks. Both possible explanations - eligibility and vigilance

451 have at least some support in the form of previous correlations with dACC activity.

452 We have proposed that this task satisfies an operational definition of curiosity

453 (Wang \& Hayden, 2018; Wang et al., 2018; Wang and Hayden, 2019). An influential

454 theory of curiosity holds that the demand for information is often driven by an

455 information gap (Golman \& Loewenstein, 2015; Gottlieb et al., 2013; Kang et al., 2009;

456 Loewenstein, 1994; Golman \& Loewenstein, 2018; Loewenstein, 1994). That is, a 
457 decision-maker's assignment of value to an informative option is caused in part by a

458 disparity between desired and actual knowledge. In this view, lack of information is a

459 drive state that can be sated by information. Consumption of information is rewarding

460 and lack of it - when desired - is aversive or at least dystonic. The information gap is the

461 central theoretical structure linking curiosity to psychology and ultimately to

462 neuroscience (Golman \& Loewenstein, 2018; Gottlieb \& Oudeyer, 2018; Kidd \&

463 Hayden, 2016; Marvin \& Shohamy, 2016; van Lieshout et al., 2018). Our results suggest

464 that the information gap would have a specific and anatomically localized set of

465 correlates, and that this set includes dACC and not OFC.

466 Our results have some bearing on debates about the ultimate nature of the dACC.

467 The function of this region has long been linked to both monitoring and executive

468 control, as well as to core economic functions (Heilbronner \& Hayden, 2016; Morecraft

469 \& Van Hoesen, 1998; Shenhav et al., 2013). Our work is most directly associated with

470 theories suggesting it is a general-purpose monitor and controller. For example, past work

471 suggests that dACC monitors conflict, reward outcomes, and other factors that lead to

472 control (Alexander \& Brown, 2011; Azab \& Hayden, 2018; Botvinick et al., 1999;

473 Shenhav et al., 2013; Shenhav et al., 2017; Hillman \& Bilkey, 2010; Widge et al., 2019).

474 Our results, then, suggest a tentative link between executive control and information-

475 seeking, one that has been generally under-appreciated in the curiosity literature. In

476 particular, they suggest that curiosity may serve be part of a larger tradeoff that involves

477 efficient allocation of cognitive resources. 
478 Functional neuroanatomy - the identification of region-specific functions is an

479 important goal of cognitive neuroscience. Some cognitive functions related to economic

480 choice appear to be broadly distributed (Cisek \& Kalaska, 2010; Hunt \& Hayden, 2017;

481 Vickery et al., 2011; Yoo \& Hayden, 2018). Our work here, however, indicates that there

482 is what appears to be a qualitative difference between OFC and AACC (Kennerley et al.

483 2011; Rudebeck et al., 2006; Hunt et al., 2018). Because we were only able to record in

484 two regions it is unclear what the full meaning of this difference is - one possibility is that

485 monitoring is a specialized cingulate function. Another possibility is that OFC is

486 specialized. Indeed, it has been proposed that OFC encodes a cognitive map of the state

487 space for the currently relevant task but is not directly involved in changing behavior

488 (Schuck et al., 2016; Wikenheiser \& Schoenbaum, 2016; Wilson et al., 2014). If so, then

489 it would not be involved in driving the state change or in keeping track of the

490 environmental variables for potential state update. Our data suggest that dACC is a strong

491 candidate for these functions, and may thus play a complementary role to OFC in this

492 process. 
494

495

496

497

498

499

500

501

502

503

504

505

506

507

508

509

510

511

512

513

514

515

516

517

518

519

520

521

522

523

524

525

526

527

528

529

530

531

532

533

534

535

536

537

\section{MATERIALS AND METHODS}

\section{Electrophysiological Techniques}

Two male rhesus macaques (Macaca mulatta) served as subjects. All procedures were approved by the University Committee on Animal Resources at the University of Rochester and were designed and conducted in compliance with the Public Health Service's Guide for the Care and Use of Animals. In this manuscript, we discuss two related datasets, one from $\mathrm{dACC}$ (the focal dataset) and one from OFC (the comparator dataset). The same subjects were used for both studies; OFC data were collected first and the dACC dataset was collected soon afterwards using the same recording methods .

A Cilux recording chamber (Crist Instruments) was placed over the prefrontal cortex, overlying both area 24 of dACC (as defined in Heilbronner and Hayden, 2016a). This is the same region used in our past studies, e.g. Hayden et al., 2011; Hayden et al., 2009. We also recorded in area 13 of OFC (Figure 1B; this is the same region used in these subjects in our past studies, for example Wang and Hayden, 2017 and Sleezer et al., 2016). Position was verified by magnetic resonance imaging with the aid of a Brainsight system (Rogue Research Inc.). Neuroimaging was performed at the Rochester Center for Brain Imaging, on a Siemens 3T MAGNETOM Trio Tim using $0.5 \mathrm{~mm}$ voxels.

Single electrodes (Frederick Haer \& Co., impedance range 0.8 to $4 \mathrm{mohm}$ ) were lowered using a microdrive (NAN Instruments) until waveforms were isolated. Action potentials were isolated on a Plexon system (Plexon, Inc). Neurons were selected for study solely based on the quality of isolation. All collected neurons for which we managed to obtain at least 500 trials were analyzed. Eye position was sampled at 1,000 $\mathrm{Hz}$ by an infrared eye-monitoring camera system (SR Research). Stimuli were controlled by a computer running MATLAB (Mathworks) with Psychtoolbox and Eyelink Toolbox. A standard solenoid valve controlled the duration of juice delivery. The relationship between solenoid open time and juice volume was established and confirmed before, during, and after recording.

\section{Information tradeoff task}

Two offers were presented in sequence on each trial. The first offer appeared for $500 \mathrm{~ms}$, followed by a $250 \mathrm{~ms}$ blank period; a second option appeared for $500 \mathrm{~ms}$ followed by a $250 \mathrm{~ms}$ blank period. Every trial had one informative and one uninformative option. The order of presentation (informative vs. uninformative) and location of presentation (info-on-left vs. info-on-right) varied randomly by trial. The offered water amount varied randomly for each option ( 75 to $375 \mu \mathrm{L}$ water in $15 \mu \mathrm{L}$ increments). $70 \%$ of trials were standard trials; for the OFC dataset, $100 \%$ of trials were standard trials. The remaining trials were variable probability trials; these were interleaved randomly with standard trials.

Each offer was represented by a rectangle 300 pixels tall and 80 pixels wide (11.35 degrees of visual angle tall and 4.08 degrees wide). On standard trials, all options offered a $50 \%$ probability of gamble win, to be delivered 2.25 seconds after the choice. Informative gambles (cyan rectangle) indicated that the subject would see a $100 \%$ valid cue immediately after choice indicating whether the gamble was won or lost. 

appear immediately after choice. Valid and invalid cues were physically identical (green and red circles inscribed on the chosen rectangle). Each offer contained an inner white rectangle. The height of this rectangle linearly scaled with the water amount to be gained in the case of a gamble win. Offers were separated from the fixation point by 550 pixels (27.53 degrees). Subjects were free to fixate upon the offers (and almost always did so). After the offers, a central fixation spot appeared. Following $100 \mathrm{~ms}$ fixation, both offers reappeared simultaneously and the animal chose one by shifting gaze to it. Then the 2.25 s delay began, and the cue was immediately displayed. Any reward was delivered after this delay. All trials were followed by a $750 \mathrm{~ms}$ inter-trial interval (ITI) with a blank screen. Previous training history for these subjects at the time of recording included a full session (several months) with this task, two types of foraging tasks (Blanchard \& Hayden, 2014; Hayden et al., 2011), three gambling/choice tasks (Farashahi et al., 2018; Heilbronner \& Hayden, 2016b; Pirrone et al., 2018), and an attentional task (similar to the one used in Hayden \& Gallant, 2013).

\section{Indifference point}

We identified when subjects chose informative and non-informative options with equal probability (50\%-50\%) and then calculated the difference in stakes (as in water amount) between the two options. We found that non-informative would have to have larger stakes than informative ones and this number is $76 \mu \mathrm{l}$ for subject B and $51 \mu \mathrm{l}$ for subject $\mathrm{H}$. Therefore, the information equates to $76 \mu \mathrm{l}$ of juice reward for subject $\mathrm{B}$ and $51 \mu \mathrm{l}$ for subject $H$.

\section{Identifying information-seeking and information-averse trials}

For the pre-trial analysis, we divided all trials into three categories, (1) ones that were more information-seeking than average (information-seeking trials), (2) ones that were less information-seeking than average (information-averse trials), and (3) ones for which we could not assign information-seeking with any confidence (neutral trials). First, we computed an equivalent value for the uninformative option by adding a session-wide average value of information for that subject (i.e. $76 \mu$ l for subject B and $51 \mu \mathrm{l}$ for subject $\mathrm{H}$ ). In effect, this means we computed the average information-seekingness of the session and then divided trials into ones that were more or less information-seeking than would be predicted given the average. Trials were placed into the first category if the subject chose the informative option and its value was less than the equivalent value of the uninformative option. Trials were placed into the second category if the subject chose the uninformative option and its equivalent value was less than the value of the informative option. Note that in many trials, the choice did not provide information germane to this question, and these were place into a third class. For example, if the informative option had a value greater than that of the uninformative one, the subject's choice would not be classifiable.

\section{Model comparison}

581 We used AIC weights to conduct model comparison and select the better fitting model. 
For model comparison, AIC weights were calculated as following:

$$
w_{i}(A I C)=\frac{\exp \left(-\frac{1}{2}\left(A I C_{i}-A I C_{\min }\right)\right)}{\sum_{r=1}^{m} \exp \left(-\frac{1}{2}\left(A I C_{r}-A I C_{\text {min }}\right)\right)},(i=1,2, \ldots, m) .
$$

584

$\mathrm{W}_{i}$ is the probability of a model $\mathrm{M}_{i}$ being the one, among all $m$ candidate models that is

585

586 closest to the true data-generating model (Burnham \& Anderson, 2010). 
Alexander, W. H., \& Brown, J. W. (2011). Medial prefrontal cortex as an action-outcome predictor. Nature Neuroscience, 14(10), 1338-1344. http://doi.org/10.1038/nn.2921

Azab, H., \& Hayden, B. Y. (2017). Correlates of decisional dynamics in the dorsal anterior cingulate cortex. PLoS Biology, 15(11), e2003091.

Azab, H., \& Hayden, B. Y. (2018). Correlates of economic decisions in the dorsal and subgenual anterior cingulate cortices. The European Journal of Neuroscience, 47(8), 979-993.

Blanchard, T. C., \& Hayden, B. Y. (2014). Neurons in Dorsal Anterior Cingulate Cortex Signal Postdecisional Variables in a Foraging Task, 34(2), 646-655. http://doi.org/10.1523/JNEUROSCI.3151-13.2014

Blanchard, T. C., Hayden, B. Y., \& Bromberg-Martin, E. S. (2015). Orbitofrontal cortex uses distinct codes for different choice attributes in decisions motivated by curiosity. Neuron, 85(3), 602-614. http://doi.org/10.1016/j.neuron.2014.12.050

Botvinick, M., Nystrom, L. E., Fissell, K., Carter, C. S., \& Cohen, J. D. (1999). Conflict monitoring versus selection-for-action in anterior cingulate cortex. Nature Publishing Group, 402(6758), 179.

Bromberg-Martin, E. S., \& Hikosaka, O. (2009). Midbrain Dopamine Neurons Signal Preference for Advance Information about Upcoming Rewards. Neuron, 63(1), 119 126. http://doi.org/10.1016/j.neuron.2009.06.009

Bromberg-Martin, E. S., Matsumoto, M., \& Hikosaka, O. (2010). Dopamine in motivational control: rewarding, aversive, and alerting. Neuron, 68(5), 815-834. http://doi.org/10.1016/j.neuron.2010.11.022

Cervera, R. L., Wang, M. Z., \& Hayden, B. (2020). Curiosity from the Perspective of Systems Neuroscience. PsychArxiv.

Cisek, P., \& Kalaska, J. F. (2010). Neural mechanisms for interacting with a world full of action choices. Annual Review of Neuroscience, 33, 269-298.

David, S. V., \& Hayden, B. Y. (2012). Neurotree: A collaborative, graphical database of the academic genealogy of neuroscience. PloS one, 7(10).

Farashahi S, Azab H, Hayden B, Soltani A. (2018) On the flexibility of basic risk attitudes in monkeys. J. Neurosci. 38, 4383 - 4398. (doi:10.1523/jneurosci. 226017.2018)

Golman, R., \& Loewenstein, G. (2015). Curiosity, Information Gaps, and the Utility of Knowledge. SSRN Electronic Journal. http://doi.org/10.2139/ssrn.2149362

Golman, R., \& Loewenstein, G. (2018). Information gaps: A theory of preferences regarding the presence and absence of information. Decision, 5(3), 143.

Gottlieb, J., \& Oudeyer, P.-Y. (2018). Towards a neuroscience of active sampling and curiosity. Nature Reviews. Neuroscience, 1.

Gottlieb, J., Oudeyer, P.-Y., Lopes, M., \& Baranes, A. (2013). Information-seeking, curiosity, and attention: computational and neural mechanisms. Trends in Cognitive Sciences, 17(11), 585-593. http://doi.org/10.1016/j.tics.2013.09.001

Hayden, B. Y., \& Gallant, J. L. (2013). Working memory and decision processes in visual area v4. Frontiers in Neuroscience, 7, 18. 
631

632

633

634

635

636

637

638

639

640

641

642

643

644

645

646

647

648

649

650

651

652

653

654

655

656

657

658

659

660

661

662

663

664

665

666

667

668

669

670

671

672

673

674

http://doi.org/10.3389/fnins.2013.00018

Hayden, B. Y., Pearson, J. M., \& Platt, M. L. (2011). Neuronal basis of sequential foraging decisions in a patchy environment. Nature neuroscience, 14(7), 933.

Hayden, B. Y., Heilbronner, S. R., Pearson, J. M., \& Platt, M. L. (2011). Surprise signals in anterior cingulate cortex: neuronal encoding of unsigned reward prediction errors driving adjustment in behavior. Journal of Neuroscience, 31(11), 4178-4187.

Hayden, B. Y., Pearson, J. M., \& Platt, M. L. (2009). Fictive reward signals in the anterior cingulate cortex. science, 324(5929), 948-950.

Heilbronner, S. R. (2017). Modeling risky decision-making in nonhuman animals: shared core features. Current opinion in behavioral sciences, 16, 23-29.

Heilbronner, S. R., \& Hayden, B. Y. (2016a). Dorsal Anterior Cingulate Cortex: A Bottom-Up View. Annual Review of Neuroscience, 39(1), annurev-neuro-070815013952. http://doi.org/10.1146/annurev-neuro-070815-013952

Heilbronner, S. R., \& Hayden, B. Y. (2016b). The description-experience gap in risky choice in nonhuman primates. Psychonomic Bulletin \& Review, 23(2), 593-600. http://doi.org/10.3758/s13423-015-0924-2

Hillman, K. L., \& Bilkey, D. K. (2010). Neurons in the rat anterior cingulate cortex dynamically encode cost-benefit in a spatial decision-making task. Journal of Neuroscience, 30(22), 7705-7713.

Hunt, L. T., \& Hayden, B. Y. (2017). A distributed, hierarchical and recurrent framework for reward-based choice. Nature Reviews. Neuroscience, 18(3), 172.

Hunt, L. T., Malalasekera, W. N., de Berker, A. O., Miranda, B., Farmer, S. F., Behrens, T. E., \& Kennerley, S. W. (2018). Triple dissociation of attention and decision computations across prefrontal cortex. Nature neuroscience, 21(10), 1471-1481.

Jepma, M., Verdonschot, R. G., Van Steenbergen, H., Rombouts, S. A., \& Nieuwenhuis, S. (2012). Neural mechanisms underlying the induction and relief of perceptual curiosity. Frontiers in Behavioral Neuroscience, 6, 5.

Kang, M. J., Hsu, M., Krajbich, I. M., Loewenstein, G., McClure, S. M., Wang, J. T.-Y., \& Camerer, C. F. (2009). The wick in the candle of learning: Epistemic curiosity activates reward circuitry and enhances memory. Psychological Science, 20(8), 963973.

Kennerley, S. W., Behrens, T. E. J., \& Wallis, J. D. (2011). Double dissociation of value computations in orbitofrontal and anterior cingulate neurons. Nature Publishing Group, 14(12), 1581-1589. http://doi.org/10.1038/nn.2961

Kolling, N., Behrens, T. E., Mars, R. B., \& Rushworth, M. F. (2012). Neural mechanisms of foraging. Science, 336(6077), 95-98.

Kidd, C., \& Hayden, B. Y. (2016). The Psychology and Neuroscience of Curiosity. Neuron, 88(3), 449-460. http://doi.org/10.1016/j.neuron.2015.09.010

Laughlin, S. B., van Steveninck, R. R. de R., \& Anderson, J. C. (1998). The metabolic cost of neural information. Nature Neuroscience, $1(1), 36$.

Loewenstein, G. (1994). The psychology of curiosity: A review and reinterpretation. Psychological Bulletin, 116(1), 75.

Marvin, C. B., \& Shohamy, D. (2016). Curiosity and reward: Valence predicts choice and information prediction errors enhance learning. Journal of Experimental Psychology: 
675

676

677

678

679

680

681

682

683

684

685

686

687

688

689

690

691

692

693

694

695

696

697

698

699

700

701

702

703

704

705

706

707

708

709

710

711

712

713

714

715

716

717

718
General, 145(3), 266.

Morecraft, R. J., \& Van Hoesen, G. W. (1998). Convergence of limbic input to the cingulate motor cortex in the rhesus monkey. Brain Research Bulletin, 45(2), 209232.

Pirrone, A., Azab, H., Hayden, B. Y., Stafford, T., \& Marshall, J. A. R. (2018). Evidence for the speed-value trade-off: Human and monkey decision making is magnitude sensitive. Decision, 5, 129-142. doi 10.1037/dec0000075

Roper, K. L. E. A. (1999). Observing Behavior in Pigeons: The Effect of Reinforcement Probability and Response Cost Using a Symmetrical Choice Procedure, 1-20.

Rudebeck, P. H., Buckley, M. J., Walton, M. E., \& Rushworth, M. F. S. (2006). A role for the macaque anterior cingulate gyrus in social valuation. Science, 313(5791), 1310-1312. http://doi.org/10.1126/science.1128197

Schuck, N. W., Cai, M. B., Wilson, R. C., \& Niv, Y. (2016). Human Orbitofrontal Cortex Represents a Cognitive Map of State Space. Neuron, 91(6), 1402-1412. http://doi.org/10.1016/j.neuron.2016.08.019

Shenhav A, Musslick S, Lieder F, et al. (2017). Toward a Rational and Mechanistic Account of Mental Effort. Annu Rev Neurosci. 40: 99-124.

Shenhav, A., Botvinick, M. M., \& Cohen, J. D. (2013). The expected value of control: an integrative theory of anterior cingulate cortex function. Neuron, 79(2), 217-240.

Sleezer, B. J., Castagno, M. D., \& Hayden, B. Y. (2016). Rule encoding in orbitofrontal cortex and striatum guides selection. Journal of Neuroscience, 36(44), 11223-11237.

Smith, E. H., Horga, G., Yates, M. J., Mikell, C. B., Banks, G. P., Pathak, Y. J., ... \& Sheth, S. A. (2019). Widespread temporal coding of cognitive control in the human prefrontal cortex. Nature neuroscience, 1-9.

Strait, C. E., Blanchard, T. C., \& Hayden, B. Y. (2014). Reward value comparison via mutual inhibition in ventromedial prefrontal cortex. Neuron, 82(6), 1357-1366. http://doi.org/10.1016/j.neuron.2014.04.032

van Lieshout, L. L., Vandenbroucke, A. R., Müller, N. C., Cools, R., \& de Lange, F. P. (2018). Induction and relief of curiosity elicit parietal and frontal activity. Journal of Neuroscience, 38(10), 2579-2588.

Vickery, T. J., Chun, M. M., \& Lee, D. (2011). Ubiquity and specificity of reinforcement signals throughout the human brain. Neuron, 72(1), 166-177.

Wang, M. Z., \& Hayden, B. Y. (2019). Monkeys are curious about counterfactual outcomes. Cognition, 189, 1-10.

Wang, M. Z., \& Hayden, B. Y. (2017). Reactivation of associative structure specific outcome responses during prospective evaluation in reward-based choices. Nature Communications, 8 , 15821. http://doi.org/10.1038/ncomms15821

White, J. K., Bromberg-Martin, E. S., Heilbronner, S. R., Zhang, K., Pai, J., Haber, S. N., \& Monosov, I. E. (2019). A neural network for information seeking. Nature communications, 10(1), 1-19.

Widge, A. S., Heilbronner, S. R., \& Hayden, B. Y. (2019). Prefrontal cortex and cognitive control: new insights from human electrophysiology. F1000Research, 8.

Wikenheiser, A. M., \& Schoenbaum, G. (2016). Over the river, through the woods: cognitive maps in the hippocampus and orbitofrontal cortex. Nature Reviews. 
719 Neuroscience, 17(8), 1-11. http://doi.org/10.1038/nrn.2016.56

720 Wilson, R. C., Takahashi, Y. K., Schoenbaum, G., \& Niv, Y. (2014). Orbitofrontal cortex

$721 \quad$ as a cognitive map of task space. Neuron, 81(2), 267-279.

722 http://doi.org/10.1016/j.neuron.2013.11.005

723 Yoo, S. B. M., \& Hayden, B. Y. (2018). Economic choice as an untangling of options

724 into actions. Neuron, 99(3), 434-447.

725 